\title{
DA PESQUISA À PRÁTICA DE ENFERMAGEM APLICANDO 0 MODELO DE ADAPTAÇÃO DE ROY
}

\author{
From research to Nursing practice applying the Roy Adaptation Model \\ De la investigación a la práctica de enfermería mediante la aplicación del Modelo de \\ Adaptación de Roy
}

Sónia Margarida Santos Coelho ${ }^{1}$

Isabel Margarida Dias Monteiro Mendes²

\begin{abstract}
RESUMO
Os modelos e as teorias de enfermagem contribuem para o desenvolvimento do conhecimento em enfermagem, guiando as investigações no sentido do desenvolvimento da disciplina e, consequentemente, da prática. De acordo como o Modelo de Adaptação de Roy, a pessoa é vista como um ser adaptável com mecanismos que permitem a adaptação de comportamentos em resposta aos estímulos ambientais. De fato, a pessoa é o enfoque central dos cuidados de enfermagem, visando-se sempre a melhoria dos cuidados prestados baseados em investigação. A metodologia do Modelo de Adaptação de Roy baseia-se na aplicabilidade do processo de enfermagem, facilitando aos enfermeiros a coleta de dados, estabelecimento de objetivos e diagnósticos de enfermagem, a determinação de intervenções de enfermagem e a posterior avaliação do processo. Assim o objetivo desta pesquisa é realizar uma revisão crítica da aplicação do Modelo Adaptação de Roy aplicado à prática e à pesquisa em Enfermagem.
\end{abstract}

Palavras-chave: Teoria de Enfermagem. Modelo de adaptação. Enfermagem.

\begin{abstract}
The models and theories of nursing contribute to the development of nursing knowledge, guiding the research towards the development of the discipline and practice accordingly. According to the Roy Adaptation Model, the person has to be seen as an adaptive mechanism that allows the adaptation of behavior in response to environmental stimuli. In fact, the person is the central focus of nursing care, always aiming at the improvement of care based on research. The methodology of the Roy Adaptation Model is based on the applicability of the nursing process, making it easier for nurses to collect data, establish goals and nursing diagnoses, the determination of nursing interventions and the subsequent evaluation process. Thus the objective of this research is to critically review the application of the Roy Adaptation Model applied into the practice and nursing research.
\end{abstract}

Keywords: Nursing Theory. Adaptation Model. Nursing

\section{Resumen}

Los modelos y teorías de enfermería contribuyen para el desarrollo del conocimiento en Enfermería, guiando las investigaciones para el desarrollo de la disciplina y, consecuentemente, de la práctica. De acuerdo con el Modelo de Adaptación de Roy se ve la persona como un mecanismo de adaptación que permite el ajuste del comportamiento en respuesta a estímulos ambientales. De hecho, la persona es el foco central de la atención de enfermería, buscando siempre el mejoramiento de la atención basada en la investigación. La metodología del Modelo de Adaptación de Roy se basa en la aplicación del proceso de enfermería, lo que facilita a las enfermeras para recopilar datos, establecer metas y diagnósticos de enfermería, la determinación de las intervenciones de enfermería y el proceso de evaluación posterior. Así, el objetivo de esta investigación es una revisión crítica de la aplicación del Modelo de Adaptación de Roy empleado para la práctica y la investigación en enfermería.

Palabras Clave: Teoría de Enfermería. Modelo de Adaptación. Enfermería

\footnotetext{
'Enfermeira no Agrupamento de Centros de Saúde Baixo Mondego III - Bussaco-Atlântico (Administração Regional de Saúde do Centro - Portugal); Mestre em Enfermagem; aluna da Pós-Licenciatura de Enfermagem de Saúde Materna e Obstetrícia da Escola Superior de Enfermagem de Coimbra.E-mail: smargaridacoelho@gmail.com; ²Enfermeira, Especialista em Enfermagem de Saúde Materna e Obstetrícia, Mestre em Saúde Pública e Doutora em Ciências de Enfermagem. Professora Coordenadora da área científica de Saúde Materna e Obstetrícia na Escola Superior de Enfermagem de Coimbra - Portugal Doutora em Enfermagem E-mail: isabelmendes@esenfc.pt
} 


\section{INTRODUCÃO}

Os conceitos são centrais, tanto para o desenvolvimento de modelos conceptuais quanto de teorias, permitindo encarar a investigação de forma mais particular. Os modelos conceituais apresentam maior nível de abstração comparativamente às teorias, eles tentam explicar globalmente os fenômenos, assegurando múltiplas possibilidades para a investigação e para a prática. 0 modelo conceitual permite reagrupar os conceitos pertinentes de forma a caracterizar ou descrever os fenômenos estudados, ${ }^{1}$ pretendendo ser representações da realidade da prática de enfermagem. ${ }^{2}$

Os profissionais de enfermagem têm investido muito no desenvolvimento de teorias e modelos de enfermagem, no sentido da melhoria dos cuidados prestados aos doentes através da educação, da prática e da investigação. No entanto, para o crescimento e desenvolvimento da disciplina e da profissão, é necessário que estes passem da formação nas escolas de enfermagem para a implementação na prática. $^{3}$

0 produto da investigação pode resultar no desenvolvimento de uma teoria pela qual a prática de enfermagem seja orientada. A teoria confere significado ao conhecimento de forma a melhorar a prática, descrevendo, explicando e antevendo os fenômenos. Os métodos sistematicamente desenvolvidos estimulam o pensamento crítico e a tomada de decisão na prática profissional de enfermagem; consequentemente, a teoria conduz à autonomia profissional, orientando a prática, o ensino e a formação da enfermagem. ${ }^{4}$ As teorias de Enfermagem começaram a emergir na década de 50 , e muitas teorias surgiram nos anos 70 à procura de explicação entre os fatos e os eventos naturais para o estabelecimento da enfermagem como ciência. A enfermagem vem ganhando espaço como uma disciplina importante na área de saúde, com o crescimento da pesquisa e uso das teorias, enquanto desempenha importante papel na promoção, prevenção e reabilitação na área da saúde. ${ }^{5}$

Partindo da sua experiência como enfermeira pediátrica, Roy considera o conceito de adaptação como um eixo orientador para a prática de enfermagem; esta teórica entende-o como um processo e resultado através do qual pessoas sensíveis e pensantes, enquanto indivíduos ou grupos, utilizam a consciência e a escolha para criar a integração humana e ambiental. ${ }^{6} \mathrm{~A}$ enfermagem fundamenta o seu conhecimento baseandose no método científico para orientar a prática dos profissionais de enfermagem, facilitando a sistematização do processo de cuidar; no entanto, existem modelos teóricos que auxiliam a sistematização de cuidar clinicamente em enfermagem, nomeadamente o Modelo de Adaptação de Roy (MAR). ${ }^{7}$

\section{O MODELO DE ADAPTAÇÃO DE ROY}

0 desafio para os profissionais de enfermagem deve passar pela melhoria da qualidade dos cuidados de enfermagem prestados a indivíduos e grupos da comunidade, assim como promover o seu reconhecimento enquanto disciplina profissional autônoma; para tal, é necessária a produção e consolidação do conhecimento. Este conhecimento é reflectido através dos modelos conceituais e teorias de enfermagem, um produto da observação na pesquisa, prática e investigação filosófica de seus autores. ${ }^{8}$

Os modelos de enfermagem são descrições da prática de enfermagem que se expressam utilizando os designados metaparadigmas de enfermagem, os conceitos de enfermagem, pessoa, ambiente e saúde.

Roy entende a Enfermagem como uma profissão dos cuidados de saúde que se centra nos processos de vida humanos, enfatizando a promoção da saúde aos indivíduos, grupos e sociedade como um todo, sendo que a ciência e a prática expande a capacidade de adaptação e melhora a transformação ambiental da pessoa. A pessoa - indivíduo, família, organizações, comunidades ou sociedade - como um todo encontra-se exposta a uma série de circunstâncias, condições ou influências que rodeiam e afetam o desenvolvimento de pessoas ou grupos, sendo que 0 ambiente em mudança estimula as pessoas a dar respostas de adaptação. 0 ambiente é considerado como todas as circunstâncias, condições e influências que rodeiam e afetam o comportamento da pessoa. A pessoa saudável não está livre de situações inevitáveis como a morte, a doença, a infelicidade ou o estresse, mas a capacidade de lidar com estas situações deve ser a mais competente possível. A saúde é um reflexo de adaptação da interação entre pessoa e ambiente. . $-3,6,9^{-1}$

Callista Roy, no seu Modelo de Adaptação, considera o objetivo da enfermagem a promoção da adaptação dos indivíduos e grupos nos quatro modos de adaptação (modo adaptativo: físico-fisiológico, identidade de autoconceito, interdependência e desempenho de papel), contribuindo assim para a saúde, a qualidade de vida e a morte com dignidade.

0 modo de adaptação físico-fisiológico está associado à forma como a pessoa responde como ser físico aos estímulos do ambiente, sendo o comportamento a manifestação das atividades fisiológicas do organismo. As cinco necessidades básicas de integridade fisiológica são oxigenação, nutrição, eliminação, atividade e repouso e proteção. Os outros modos de adaptação são modos psicossociais: autoconceito, interdependência e desempenho de papel. 0 autoconceito envolve especificamente os aspectos psicológicos e espirituais do sistema humano. É composto pelo ser físico, que envolve a imagem corporal, pelo ser pessoal, que engloba a autoconsciência, o autoideal ou expectativa, e pelo ser ético, moral e espiritual. 0 modo de adaptação desempenho de papel refere-se aos papéis que cada pessoa desempenha na sociedade no sentido da preservação da 
integridade social, isto é, saber quem se é em relação aos outros.

As pessoas desempenham papéis primários (determinam a maioria dos comportamentos tidos pela pessoa em uma fase da vida e são determinados pela idade, sexo ou estádio de desenvolvimento), secundários (assumidos pela pessoa para cumprir a tarefa associada a um estádio de desenvolvimento ou a um papel primário) ou terciários (são de natureza temporária e livremente escolhidos pelo indivíduo), que podem ser levados a cabo por comportamentos instrumentais, que são aqueles em que há um desempenho real e físico do comportamento, ou comportamentos expressivos que envolvem as emoções, sentimentos atitudes de uma pessoa perante um papel ou desempenho. Por último, o modo de adaptação interdependência centra-se nas relações próximas entre as pessoas. $^{3,9}$

No MAR as pessoas são consideradas como sistemas abertos, os quais mantêm interação contínua com seus ambientes, ocorrendo mudanças internas e externas. As pessoas estão constantemente sujeitas a estímulos que exigem respostas, que podem ser respostas adaptativas se promovem a integridade em termos dos objetivos dos sistemas humanos como a sobrevivência, o crescimento, a reprodução, formação e a integração da pessoa no meio ambiente, ou ineficazes se não contribuem em termos dos objetivos do sistema humano. ${ }^{2-}$ 3,6,9 Estímulo é entendido como tudo o que desencadeia uma resposta - o foco de interação entre sistema humano e ambiente, originando-se do ambiente externo (estímulo externo) ou do ambiente interno (estímulo interno), sendo descritos três tipos de estímulos que interagem com as pessoas: focal, contextual e residual. Estímulos focais são aqueles estímulos internos ou externos que confrontam imediatamente a pessoa. Estímulos contextuais são todos os outros estímulos, presentes na situação, contribuindo para o efeito do estímulo focal, isto é, todos os fatores ambientais internos ou externos que se apresentam à pessoa, que mesmo não sendo o centro da atenção, podem influenciar a forma como a pessoa reage aos estímulos focais. Estímulos residuais são fatores ambientais internos ou externos, cujos efeitos na situação não se encontrem claros ou, simplesmente, não possam ser avaliados..$^{2-3,5-6}$

Os processos centrais da adaptação, segundo o MAR, são o subsistema regulador e o cognitivo. 0 subsistema regulador inclui os canais neuroquímicos e endócrinos que a pessoa aciona mediante uma resposta quase automática e inconsciente. 0 subsistema cognitivo processa informação perceptual, a aprendizagem, os juízos e as emoções, para que a pessoa possa realizar a sua resposta. ${ }^{2-3}$

\section{O PROCESSO DE ENFERMAGEM E 0 MODELO DE ADAPTAÇÃO DE ROY}

prática de enfermagem, sistematiza a assistência de enfermagem, isto é, indica um determinado método ou modo de fazer fundamentado em um sistema de valores e crenças morais e no conhecimento técnico-científico da área. ${ }^{10}$

A orientação da prática clínica partindo do MAR é possível, uma vez que este consegue orientar o processo de enfermagem (ver Figura 1).

Figura 1. 0 Modelo de Adaptação de Roy e o Processo de Enfermagem (adaptado de Gary, 2001)

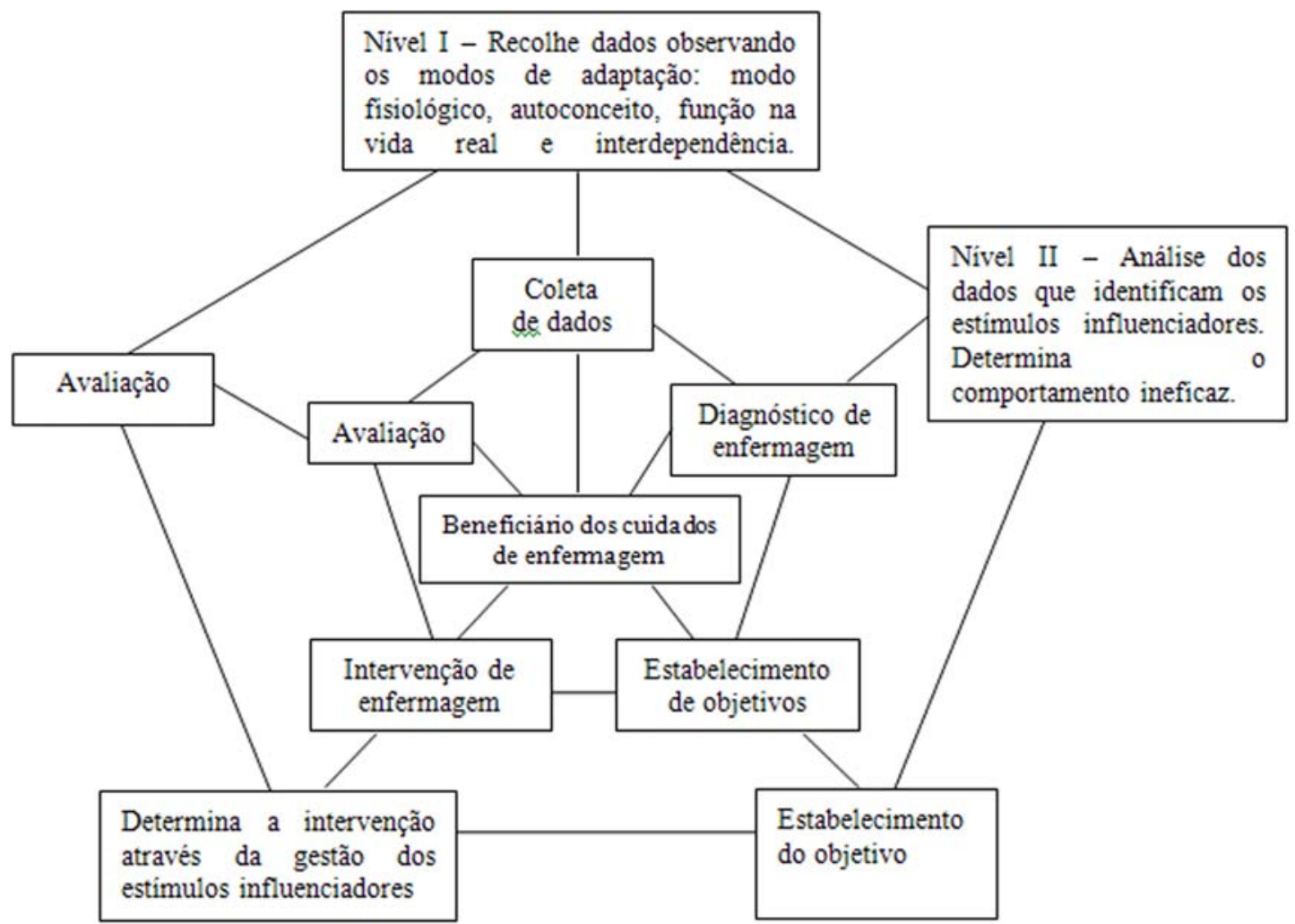


0 processo de enfermagem pode ser visualizado de acordo com o MAR. Os componentes do processo são: a avaliação do comportamento, a avaliação de estímulos, diagnóstico de enfermagem, estabelecimento de objetivos, intervenção e

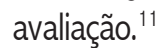

0 MAR pode ser um instrumento valioso na orientação prática dos enfermeiros no processo de enfermagem que leva à formulação de diagnósticos de enfermagem preditores das intervenções de enfermagem. 0 centro dos cuidados de enfermagem pode ser a pessoa, a família e a comunidade. 0 enfermeiro que apoie a sua prática no MAR vê o centro dos seus cuidados como coextensões com o seu ambiente físico e social, considerando as suas crenças, valores e esperanças. ${ }^{2}$

A recolha de dados do processo de enfermagem consiste no primeiro nível para a identificação dos comportamentos do beneficiário dos cuidados de enfermagem, observando os quatro modos de adaptação (o modo fisiológico, o modo de autoconceito, o modo da função na vida real e o modo de interdependência). 0 segundo item do processo de enfermagem - diagnóstico de enfermagem - corresponde ao segundo nível de avaliação que identifica os estímulos influenciadores de comportamentos ineficazes. Estes dois níveis auxiliam a profissional de enfermagem no estabelecimento de objetivos. Outro item do processo de enfermagem é a determinação das intervenções; estas devem ser encontradas através da gestão dos estímulos influenciadores do comportamento. A avaliação comum ao processo de enfermagem ao modelo poderá novamente levar o enfermeiro a uma nova coleta de dados. ${ }^{3,12}$

\section{MODELO DE ADAPTAÇÃO DE ROY NA PRÁTICA E NA INVESTIGAÇÃO}

À medida que a Enfermagem amadurece enquanto profissão, consequentemente, o profissional de enfermagem torna-se mais consciente da importância da prática baseada na teoria e das vantagens da implementação de um modelo de conceptualização da enfermagem como base para a prática. As vantagens enunciadas incluem: aumento dos cuidados de enfermagem aos utentes; avaliação e diagnósticos de enfermagem mais abrangentes e rigorosos com uma especial atenção às necessidades psicossociais dos utentes; melhoria da comunicação inter e intraprofissional e, consequentemente, níveis mais elevados de autoestima profissional; registros de enfermagem mais claros, objetivos e rigorosos. ${ }^{3}$

0 objetivo de um modelo de enfermagem é a orientação para a prática de enfermagem, fornecendo também uma perspectiva da forma a partir da qual poderemos visualizar e desenvolver o conhecimento disciplina. Este conhecimento de acordo com o MAR, pode subdividir-se em ciência básica da enfermagem e ciência clínica da enfermagem. 0 conhecimento básico consiste em compreender a adaptação das pessoas, no âmbito das suas situações de vida variadas, contemplando os processos adaptáveis da pessoa/grupo, modos adaptáveis e adaptação relacionada com a saúde. A ciência de enfermagem relaciona-se com o conhecimento básico de adaptação das pessoas para compreendê-las em situações de saúde e de doença, e o que pode ser feito para intensificar a adaptação. Esta estrutura contempla as mudanças na eficácia das atividades, as mudanças dentro e entre os modos adaptáveis e cuidados de enfermagem para promover os processos adaptáveis. ${ }^{3,11}$

0 MAR insere-se na Escola dos Resultados, centrandose nas respostas humanas nos processos de vida e no controle dos fatores que originam as necessidades de adaptação. ${ }^{15}$

Os fenômenos de interesse para pesquisa mediado pelo MAR são problemas que incluem as tentativas pessoais para atender às suas necessidades fisiológica, psicológica, espiritual, social e relacional, bem como os problemas decorrentes das tentativas feitas por grupos de satisfazer as suas necessidades coletivas para a adequação dos recursos, identidade e integridade relacional, e clareza de função. ${ }^{11}$

Os participantes na investigação MAR podem ser indivíduos ou grupos saudáveis ou com problemas de saúde agudos ou crônicos. A metodologia aplicada à pesquisa pode ser qualitativa ou quantitativa descritiva, correlacional e experimental. Os instrumentos de investigação devem refletir o foco único e intenção do MAR. ${ }^{11}$

A finalidade do MAR é aumentar o nosso conhecimento para a prática através da investigação, e orientar a educação da enfermagem na ciência e arte de enfermagem. ${ }^{3}$

0 objetivo geral da enfermagem, de acordo com Roy, é o objetivo dos cuidados de saúde: a promoção da saúde. A pessoa, enquanto metaparadigma do modelo referenciado, é entendida como um sistema de adaptação, isto é, o ser humano é descrito como um todo com partes que funcionam de acordo com algum objetivo. 0 sistema humano representa o objeto do cuidado de enfermagem e é possuidor de mecanismos cognitivos e conceptualizadores que atuam para promover a adaptação em cada um dos modos adaptáveis: fisiológico, autoconceito, função na vida real e interdependência. ${ }^{13}$

0 modelo identifica-se claramente como fenômeno a estudar as pessoas, como indivíduos ou grupos, e dá o seu contributo para o desenvolvimento do conhecimento através do fornecimento de uma perspectiva de investigação.

0 MAR é útil para a prática de enfermagem, porque esboça os aspectos da disciplina e fornece orientações para a prática, ensino e investigação $0^{6}$, oferece uma melhor percepção das situações vivenciadas, contribui para a observação dos comportamentos manifestados pelos utentes e, desta forma, auxilia na construção das intervenções de enfermagem. ${ }^{5}$

A utilização de um modelo conceitual para orientar a pesquisa em Enfermagem e/ou Prática envolve um processo composto por três etapas: ${ }^{11}$

1. Desenvolver uma compreensão detalhada do conteúdo e das diretrizes de pesquisa e prática do modelo conceptual. É necessário entender o conteúdo do modelo 
conceitual, no MAR. Este é composto de conceitos tão abstratos como estímulos ambientais, mecanismos de resistência, e modos de adaptação, sendo necessário compreender as instruções que definem amplamente cada conceito.

2. Rever a literatura existente sobre a utilização do modelo conceitual como base para a prática e para a investigação. É fundamental a revisão da literatura existente para orientar a pesquisa e a prática, podendo os conceitos centrais do modelo orientar a categorização dos resultados da pesquisa bibliográfica.

3. Construir e comunicar claramente uma estrutura conceitual, teórico-empírico acerca das temáticas de investigação e da prática.

\section{SISTEMAS DE INFORMAÇÃO EM ENFERMAGEM E MODELO DE ADAPTAÇÃO DE ROY}

As mudanças das circunstâncias ambientais podem alterar o significado do estímulo. Um estímulo pode ser contextual em um momento específico e tornar-se focal em outro, ou viceversa; como tal, as enfermeiras devem definir, conceitualizar e caracterizar os estímulos para que não surjam dúvidas na utilização do modelo teórico.

Neste campo, a Classificação Internacional para a Prática de Enfermagem é uma classificação de fenômenos, ações e resultados que pretende ser uma matriz unificadora em que as taxonomias e as classificações de enfermagem existentes se entrecruzam. ${ }^{14}$ Portanto, mesmo que um profissional de enfermagem utilize o MAR na conceitualização da prática dos seus cuidados, pode e deve utilizar a Classificação Internacional para a Prática de Enfermagem, uma vez que esta estabelece uma linguagem comum para descrever a prática de enfermagem e fornece dados que possam influenciar a formação em enfermagem, incentiva a investigação e a melhoria das práticas.

Aplicando o MAR como o apoio da Classificação Internacional para a Prática de Enfermagem, ocorrem consequências deriváveis, isto é, a forma como a teoria é utilmente utilizada influencia a prática de cuidados, e outra perspectiva não era possível de vislumbrar devido à transversalidade da classificação. 0 modelo tem um processo de enfermagem definido claramente e, assim, orienta a prática clínica de enfermagem orientando a prestação de cuidados e, consequentemente, orienta a investigação e o ensino. ${ }^{6}$

A emergência dos sistemas de informação em enfermagem veio reforçar a necessidade de desenvolvimento de classificações de enfermagem, e nesta abordagem surgiu a Classificação Internacional para a Prática de Enfermagem. A necessidade de comunicar através de uma linguagem comum, em que todos os profissionais de enfermagem atribuam os mesmos significantes aos mesmos significados, promove a evolução da profissão e de investigação em enfermagem. Os sistemas de informação em enfermagem têm que dar respostas aos modelos de enfermagem utilizados pelos profissionais; de outra forma, a utilização dos sistemas não seria aceita, não sendo eles limitadores do desenvolvimento das práticas, mas sim promotores. ${ }^{15}$

\section{DIFICULDADES NA APLICAÇÃO DO MODELO DE ADAPTAÇÃO DE ROY}

As dificuldades na aplicação do MAR podem ser identificadas na relação com a prática de enfermagem ou com a educação dos profissionais. ${ }^{2} 0$ desenvolvimento do MAR foi baseado na prática da teórica de enfermagem e, como tal, é representativo da realidade; no entanto, a utilização eficiente do modelo na prática de enfermagem encontra-se dependente do nível compreensão deste pelos enfermeiros na prática clínica. A proximidade semântica dos conceitos utilizados no modelo e no processo de enfermagem podem ser uma dificuldade para enfermeiras que não tenham estas duas entidades conceitualmente definidas.

\section{CONCLUSÃO}

A enfermagem, de acordo como o MAR, é a ciência e a prática que aumenta as capacidades adaptativas e procura transformações nas pessoas inseridas no meio ambiente. A aplicação do conceito de pessoa como sistema adaptável permite aos enfermeiros a conceitualização dos cuidados baseados na interação da pessoa com o seu ambiente.

0 MAR é uma importante ferramenta para o desenvolvimento da prática e investigação em enfermagem, proporcionando relevância social à profissão. Na prática de enfermagem permite uma boa percepção das situações, contribuindo para uma boa elaboração de diagnósticos, auxiliando na prescrição de intervenções de enfermagem; uma vez que tem o processo de enfermagem claramente definido, consegue ser útil na orientação da prática já que fornece uma visão holística do utente/doente. 0 MAR norteia a investigação no sentido de fornecer uma perspectiva orientadora da investigação; isto é, o modelo fornece várias hipóteses testáveis perante um problema. Uma vez que 0 MAR distingue a ciência de enfermagem de outras ciências na área da saúde pode ser um modelo base a ser aplicado na formação em enfermagem.

\section{REFERÊNCIAS}

1 - Fortin MF, Côté J, Filion F. Fundamentos e etapas do processo de investigação. Tradução de Nídia Salgueiro. Loures: Lusodidacta; 2009.

2 - Roy C. El modelo de adaptación de Roy en el contexto de los modelos de enfermería, con ejemplos de aplicación y dificultades. Cultura de los Cuidados. Rev Enferm Human. $20001^{\circ} / 2^{\circ}$ sem; 4 (07-08): 139 -59. 
3 - Andrews HA, Roy C. Pontos essenciais do Modelo de Adaptação de Roy. In: Roy C, Andrews, HA. Teoria da Enfermagem: o Modelo de Adaptação de Roy. Lisboa: Instituto Piaget; 2001. p. 15- 39.

4 - Tomey AM, Alligood MR. Significado da teoria para a enfermagem, enquanto disciplina e profissão. In: Tomey AM, Alligood MR. Teóricas de enfermagem e a sua obra: modelos e teorias de enfermagem. Loures: Lusociência; 2004. p. 15-34.

5 - Mesquita Melo E, Lopes MV, Carvalho Fernandes, AF, Teixeira Lima FE, Barbosa IV. Teorias de enfermagem: importância da correta aplicação dos conceitos. Enferm. Glob. [on-line]. 2009 out; [citado 2011 mar 03]; 17: [aprox. 9 telas]. Disponível em: http://www.scielo.br.

6 - Phillips KD. Irmã Callista Roy: Modelo de Adaptação. In: Tomey AM, Alligood MR. Teóricas de enfermagem e a sua obra: modelos e teorias de enfermagem. Loures: Lusociência; 2004. p. 335 - 33.

7 - Oliveira MF, Silva LF. Enfermagem em laboratório de hemodinâmica: diagnóstico e intervenção fundamentados na Teoria da Adaptação de Roy. Rev Eletr Enferm. [on- line] 2010; [citado 2011 março 18]; 12 (4): [aprox. 8 telas]. Disponível em: http://www.fen.ufg.br/revista/ v12/n4/v12n4a12.htm.

8 - Moreno-Fergusson ME, Alvarado-Garcia AM. Aplicación del Modelo de Adaptación de Callista Roy en latinoamérica: revisión de la literatura. Aquichan. [on line] 2009 jan/june; [citado 2011 mar 03]; 9(1): [aprox. 11 telas]. Disponível em http://www.scielo.br.

9 - Rodrigues DP, Pagliuca LMF, Silva RM. Modelo de Roy na enfermagem obstétrica: análise sob a óptica de Meleis. Rev Gaucha Enferm. [online]. 2004 ago; [citado 2011 mar 03]; 25(2): (aprox. 10 telas). Disponível em: http://www.scielo.br.

10 - Garcia TR, Nóbrega MML. Processo de enfermagem: da teoria à prática...Esc Anna Nery. 2009 mar; 13 (1): 188-93.

11 - Fawcett J. Using the Roy Adaptation Model to guide research and/ or practice: construction of conceptual-theoretical-empirical systems of knowledge. Aquichán. [on- line] 2009 Sep/Dec; [citado 2011 mar 03]; 9(3): [aprox. 10 telas]. Disponível em: http://www.scielo.br.

12 - Gary J. 0 Modelo de Adaptação de Roy na prática da enfermagem. In: Roy C, Andrews, HA. Teoria da enfermagem: o Modelo de Adaptação de Roy. Lisboa: Instituto Piaget; 2001. p. 483- 98.

13 - Roy C. 0 Modelo de Adaptação de Roy na investigação da enfermagem. In: Roy C, Andrews, HA. Teoria da enfermagem: o Modelo de Adaptação de Roy. Lisboa: Instituto Piaget; 2001. p. 499- 514.

14 - Conselho Internacional de Enfermeiras. Classificação Internacional para a Prática de Enfermagem (CIPE/ICNP) - Versão Beta 2. $3^{\text {a }}$ ed. Lisboa: Associação Portuguesa de Enfermeiros; 2005.

15 - Silva AAP. Sistemas de Informação em Enfermagem: uma teoria explicativa da mudança. Lisboa: Formasau - Formação e Saúde; 2006. 